\title{
FRAMEWORK AND ARCHITECTURE FOR SECURE MOBILE BUSINESS APPLICATIONS
}

\author{
Jochen Haller, Philip Robinson, Thomas Walter, Roger Kilian-Kehr
}

SAPAG, CEC Karlsruhe

[jochen.haller | philip.robinson | roger.kilian-kehr]@sap.com

DoCoMo Euro-Labs

walter@docomolab-euro.com

Abstract: With the mobile telecommunication systems (GPRS, UMTS) enterprises have the possibility to better integrate their mobile employees into their backend business processes. However, from an enterprise perspective access to security-critical information from a mobile user's device requires appropriate and often enormous management efforts. This typically ranges from advanced firewall policies to special security components such as virtual private networks. These mechanisms are not suitable for the broad range of mobile business applications that require security properties independent from the properties guaranteed by the network layer. To achieve true security for these applications an appropriate application framework is presented that facilitates the development and deployment of secure mobile business applications.

Key words: mobility, business applications, security, security policies, smart cards

\section{INTRODUCTION}

In recent years the number of mobile users has steadily increased. Companies are seeking solutions to support their mobile workforce with online access to their corporate networks and applications. Traditional solutions like firewall policies [2] or virtual private networks (VPN) [3] are rather inflexible when used in an environment with mobile users.

The original version of this chapter was revised: The copyright line was incorrect. This has been corrected. The Erratum to this chapter is available at DOI: 10.1007/978-0-387-35691-4_52 
The WiTness ${ }^{1}[4][1]$ - Wireless Trust for Mobile Business - project looks into an application layer security framework for secure mobile business applications.

\section{SECURE MOBILE BUSINESS APPLICATION}

Traditional (client-server based) business applications are based on multitier architectures where client systems communicate with several servers to process business transactions. The expansion of mobile devices and consequent mobility of users adds another level of complexity with respect to heterogeneity and number of devices representing the "client side". The notion of a secure mobile business application is therefore a move towards coping with this complexity.

The term secure implies that particular policies must be satisfied with respect to accessing critical resources (data, processes, network equipment, etc.). The enforcement of these policies may include mechanisms for assurance of mutual authentication, authorization, integrity, and confidentiality.

Mobility is enabled by the dimensional properties of devices as well as the properties of the communications media and infrastructure. Consider small devices with limited resources like processing power, display and storage communicating over heterogeneous wireless networks, for instance GSM or 802.11 WaveLan.

Applications are the way that a business' productivity and processes are technically implemented. They therefore should reflect company policies, including security policies, through their implementation.

Application layer security represents true end-to-end security, independent from underlying network security mechanisms.

Furthermore, application layer security makes the integration of security into the application design process a more realistic and conceptually feasible task. There are also specialized applications explicitly referred to as security services. The foremost of these security services is the security module whose architectural properties are also further defined in this chapter.

\footnotetext{
' IST-Programme / KA2 / AL: IST-2001-2.1.3.
} 


\section{FRAMEWORK AND ARCHITECTURE}

WiTness is concerned with a business-to-employee scenario with three identified main domains of interest: corporate domain, personal domain, and network domain.

\subsection{Corporate domain}

The corporate domain includes all systems, system components, either hard- or software required to run business applications. In particular. information provider and server systems and their respective interconnecting networks are meant. It is assumed that all connections within the corporate domain as well as every system is secure.

\subsection{Personal domain}

The counterpart of the corporate domain is the personal domain. It consists at least of a mobile device (e.g. a mobile phone) and a smart card. More complex personal domains can be generated by simply adding further (mobile) devices setting up so-called federations of client devices.

The essential requirements for federations are that communication between devices and between the federation and corporate domain runs over secured channels.

Within a federation, a smart card performs a specific role. It implements the before mentioned security module. A security module holds a set of user credentials which allow an employee to identify and authenticate with the corporate domain and to perform further security related tasks (e.g., session key generation or signing data hashes).

\subsection{Network and Operator domain}

Mobility of users and devices is best achieved and guaranteed with a wireless network infrastructure. Because all security related functions are implemented in the application itself, it is even of no relevance which security support is implemented in the network domain. Consequently. WiTness abstracts the heterogeneity of underlying networks, primarily with respect to their security provisions. 


\subsection{Core WiTness architecture}

The core architecture comprises the following elements:

- Application modules are implemented as partlets. These are either preinstalled or dynamically downloaded to mobile devices. Partlets encode the business logic of an application.

- WiTness provides implementations and supporting libraries for handling of federations, security logic and communications handling.

- Additionally, a security service is implemented in the security module. It is a stand-alone security application providing services and functions such as key generation, document signing, en- and decryption with private key, certificate management etc.

- With regards to policies and policy management, WiTness also delivers methodologies and utilities for policy management. Policies are enforced by security logic, which again is implemented in the partlets complementing the business logic.

\section{CONCLUSION}

This contribution has provided an overview of the architecture for secure mobile business applications introduced by the WiTness project. The secure mobile business application itself was introduced and the different domains - personal, corporate and network - necessary to introduce the core WiTness architecture. The final chapter deals with the actual application, its design process, implementation and finally its deployment.

\section{REFERENCES}

[1] Sheila Frankel, Demystifying the IPsec Puzzle, Artech House, 2001.

[2] William Stallings, Cryptography and Network Security - Principles and Practice - Second Edition, Prentice Hall, 1999.

[3] William Stallings, IP Security, The Internet Protocol Journal, Volume 3, Number 1, www.cisco.com/warp/public/759/ipj issues.html, March 2002.

[4]. Thomas Walter, Peter Schoo, Jochen Haller, Philip Robinson, WiTness - Wireless Trust for Mobile Business - Position Statement, PAMPAS Workshop, Royal Holloway, University of London, www.pampas.eu.org/workshop.html, September 2002.

[5] WiTness consortium, Wireless Trust for Mobile Business, IST-200132275, www.wireless-trust.org, 2002. 